\title{
Search for the signal of monotop production at the early LHC
}

\author{
Jian Wang, ${ }^{1}$ Chong Sheng Li*$^{*},{ }^{1,2}$ Ding Yu Shao, ${ }^{1}$ and Hao Zhang ${ }^{1}$ \\ ${ }^{1}$ Department of Physics and State Key Laboratory of Nuclear Physics and Technology, \\ Peking University, Beijing, 100871, China \\ ${ }^{2}$ Center for High Energy Physics, Peking University, Beijing, 100871, China
}

\begin{abstract}
We investigate the potential of the early LHC to discover the signal of monotops, which can be decay products of some resonances in models such as R-parity violating SUSY or SU(5), etc. We show how to constrain the parameter space of the models by the present data of $Z$ boson hadronic decay branching ratio, $K^{0}-\overline{K^{0}}$ mixing and dijet productions at the LHC. Then, we study the various cuts imposed on the events, reconstructed from the hadronic final states, to suppress backgrounds and increase the significance in detail. And we find that in the hadronic mode the information from the missing transverse energy and reconstructed resonance mass distributions can be used to specify the masses of the resonance and the missing particle. Finally, we study the sensitivities to the parameters at the LHC with $\sqrt{s}=7 \mathrm{TeV}$ and an integrated luminosity of $1 \mathrm{fb}^{-1}$ in detail. Our results show that the early LHC may detect this signal at $5 \sigma$ level for some regions of the parameter space allowed by the current data.

PACS numbers: $12.38 . \mathrm{Bx}, 12.60 .-\mathrm{i}, 14.65 . \mathrm{Ha}$
\end{abstract}

\footnotetext{
* Electronic address: csli@pku.edu.cn
} 


\section{INTRODUCTION}

The main tasks of the Large Hadron Collider (LHC) are to answer the fundamental questions in particle physics: whether the Higgs boson exist or not. And are there new physics beyond standard model (SM) such as supersymmetry (SUSY), extra dimension, etc, at the TeV scale? Generally, it is believed that top quark may have strong connections with new physics due to its large mass close to the scale of electroweak symmetry breaking. The production topologies of top quark pair production with or without missing transverse energy $E_{T}$ have been extensively investigated [1 [10]. However the topology of a single top and $E_{T}$, which is so-called monotop [11], has only been discussed recently [12, [13]. This signal is absent in the SM and occur in models such as R-parity violating SUSY and SU(5) as decay products of resonance production of some particles. In R-parity violating SUSY [14, a stop can be produced by the fusion of two down-type anti-quarks through the Yukawalike trilinear interaction $\lambda_{i j k}^{\prime \prime} U_{i}^{c} D_{j}^{c} D_{k}^{c}$, where $U_{i}, D_{i}$ are left-handed chiral superfields and the superscript $c$ denotes the charge conjugate, and then the stop decays into a top quark and a neutralino which could not be detected at the collider. In the SU(5) model [15], the gauge bosons $X$, in one case, can transform quarks to anti-quarks assigned to the $\mathbf{1 0}$ representation; in the other case, they couple to quarks and leptons in the $\mathbf{5}$ representation. As a result, they can be resonantly produced at hadron colliders and decay into a top and a neutrino. Therefore, any discovery of such signal imply new physics, and may help us to explore the fundamental questions mentioned above.

In this work, we propose the general model-independent renormalizable effective Lagrangian with $\mathrm{SU}(3)_{\mathrm{c}} \times \mathrm{SU}(2)_{\mathrm{L}} \times \mathrm{U}(1)_{\mathrm{Y}}$ gauge symmetry

$$
\mathcal{L}=\lambda_{S}^{i j} \epsilon^{\alpha \beta \gamma} \phi_{\alpha} \bar{d}_{i \beta R}^{c} d_{j \gamma R}+a_{S}^{i} \phi_{\alpha} \bar{u}_{i R}^{\alpha} \chi+\lambda_{V}^{i j} \epsilon^{\alpha \beta \gamma} X_{\mu, \alpha} \bar{d}_{i \beta R}^{c} \gamma^{\mu} d_{j \gamma R}+a_{V}^{i} X_{\mu, \alpha} \bar{u}_{i R}^{\alpha} \gamma^{\mu} \chi+\text { h.c. },
$$

where there is a summation over the generation indices $i, j=1,2,3$, and $\mathrm{SU}(3)_{\mathrm{c}}$ gauge indices $\alpha, \beta, \gamma=1,2,3$. The superscript $c$ denotes charge conjugation. The Dirac field $\chi$ is a singlet under the SM gauge group and manifest itself as missing energy at colliders. The scalar and vector fields $\phi$ and $X_{\mu}$ are color triplet resonances that can appear in some models, which obtain their masses at high energy scales. This Lagrangian could further be generalized, such as shown in Ref. [11, although it may not be gauge invariant any more. The free parameters in Eq. (11) are masses of the resonances and missing particle, i.e., $m_{\phi}, m_{X}$ and $m_{\chi}$, and couplings $\lambda_{S, V}^{i j}$ and $a_{S, V}^{i}$, which should be constrained by current 
precise data, and will be investigated carefully in this paper. Here, we only consider the case of scalar resonance field $\phi$, and the case of vector resonance field $X_{\mu}$ will be studied elsewhere.

The scenario of monotop production has been explored in Ref. [11], where they only consider the mode of top hadronic decay. In the case of resonant monotop production, they assume the branching fraction of $\phi \rightarrow t \chi$ equal to one and neglect the decay channel of $\phi \rightarrow \bar{d} \bar{s}$, which would lead to an overestimation of the signal. But we will take into account all decay channels of the resonance, which turns out to be very important for estimating the sensitivity to detect the signal at the LHC. Moreover, we also discuss the mode of semileptonic decay of top quark besides hadronic decay. Although the cross section of the backgrounds for semileptonic decay mode are very large, the discovery of the signal in this mode is still possible once appropriate cuts are imposed.

This paper is organized as follows. In Sec. [1], we consider the constraints on the free parameters from $Z$ hadronic decay branching ratio, $K^{0}-\overline{K^{0}}$ mixing and dijet experiments at the LHC. In Sec. [II], we investigate the signal and backgrounds of monotop production in detail and then analyze the discovery potential at the early LHC. A conclusion is given in Sec. [V].

\section{EXPERIMENT CONSTRAINTS}

The experiments have set constraints on the stop production and decay, the signal of which is similar to the monotop, in R-parity violating SUSY so far. For example, the H1 [16] and ZEUS [17] collaborations at HERA have analyzed the process of stop resonantly produced by electron-quark fusion and followed either by a direct R-parity-violating decay, or by the gauge boson decay. The process of stop pair production and decaying into dielectron plus dijet at the Tevatron is also discussed [18]. However, these results can not be converted to constraints on the parameters in our case. Here the relevant experiments, we are concerned with, are $Z$ hadronic decay branching ratio, $K^{0}-\overline{K^{0}}$ mixing and dijet production at the LHC. 

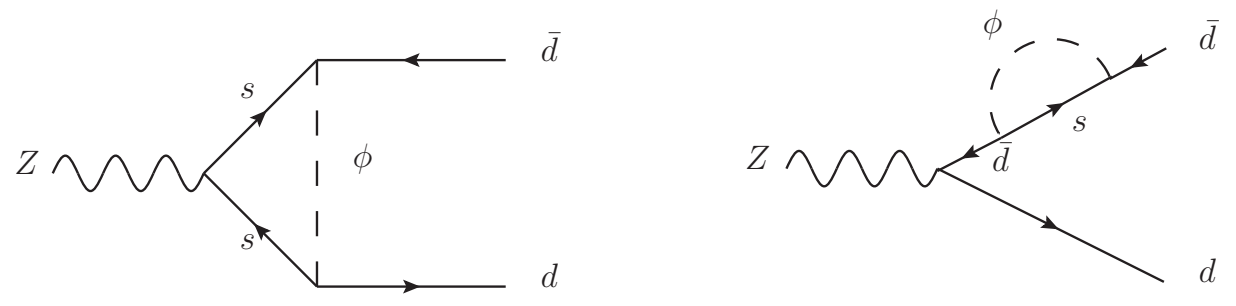

FIG. 1: Feynman diagrams for hadronic $Z$ boson decay induced by the field $\phi$.

\section{A. $Z$ hadronic decay branching ratio}

The effective Lagrangian in Eq. (1) may contribute to the branching fraction of $Z$ boson hadronic decay as shown in Fig. 1. From the precise measurement of branching fraction of $Z$ boson hadronic decay, the relevant bands on R-parity violating SUSY parameters have been

investigated in Ref. [19]. Since the quarks in the effective Lagrangian are right-handed, the couplings of right-handed quarks with $Z$ boson are modified, and thus affect the branching fraction of $Z$ boson hadronic decay.

The tree-level amplitude of $Z$ boson decaying into a pair of quarks in the SM can be parameterized as

$$
\mathcal{M}_{\mu}=g_{Z} \bar{q}\left(p_{1}\right) \gamma^{\mu}\left(a_{L}^{q} P_{L}+a_{R}^{q} P_{R}\right) q\left(p_{2}\right)
$$

where

$$
\begin{aligned}
g_{Z} & =\frac{e}{s_{W} c_{W}}, \\
a_{L}^{q} & =t_{3}^{q}-Q_{q} s_{W}^{2}, \\
a_{R}^{q} & =-Q_{q} s_{W}^{2} .
\end{aligned}
$$

After calculating the Feynman diagrams in Fig. 1, we find that the coefficient $a_{R}^{q}$ is adjusted by multiplying a factor

$$
1+\Delta_{f}=1+\frac{\lambda_{f}^{2}}{8 \pi^{2}} g(a)
$$

where $a=M_{Z}^{2} / m_{\phi}^{2}$ and $f=1,2,3$ correspond to $Z$ boson decaying into $d \bar{d}, s \bar{s}$ and $b \bar{b}$, respectively. And $\lambda_{f}$ are defined as

$$
\begin{aligned}
& \lambda_{1}^{2}=4\left[\left(\lambda_{S}^{12}\right)^{2}+\left(\lambda_{S}^{13}\right)^{2}\right], \\
& \lambda_{2}^{2}=4\left[\left(\lambda_{S}^{12}\right)^{2}+\left(\lambda_{S}^{23}\right)^{2}\right], \\
& \lambda_{3}^{2}=4\left[\left(\lambda_{S}^{13}\right)^{2}+\left(\lambda_{S}^{23}\right)^{2}\right],
\end{aligned}
$$


where we have used the fact that $\lambda_{S}^{i j}=-\lambda_{S}^{j i}$ due to the antisymmetry of the $\epsilon^{\alpha \beta \gamma}$ couplings in Eq. (11). The explicit form of function $g(a)$ is

$$
g(a)=\frac{(a-4) a-2 \log (a)((a-2) a+2 \log (a+1))-4 \mathrm{Li}_{2}(-a)}{4 a^{2}} .
$$

The ultraviolet poles of the triangle and self-energy diagrams have canceled each other, and we obtain a finite result. In this calculation, all the masses of quarks are neglected. Eq. (8) seems divergent if $a$ vanishes due to the denominator $a^{2}$. But actually we expand this result around $a=0$, and get the asymptotic form

$$
g(a)=\left(\frac{1}{9}-\frac{\log (a)}{3}\right) a+\left(\frac{\log (a)}{4}-\frac{1}{16}\right) a^{2}+\left(\frac{1}{25}-\frac{\log (a)}{5}\right) a^{3}+O\left(a^{4}\right),
$$

which vanishes obviously when taking the limit $a \rightarrow 0$. This feature guarantees the decouple of the heavy particle $\phi$ in the large $m_{\phi}$ limit.

There are two observables which can be affected by the change of coefficient $a_{R}^{q}$. One is $R_{l} \equiv \Gamma_{h} / \Gamma_{l}$, where $\Gamma_{h, l}$ are the widths of $Z$ boson decaying into hadrons and leptons, respectively. The correction to $R_{l}$ is

$$
\begin{aligned}
\delta R_{l} & =\frac{\Gamma_{h}-\Gamma_{h}^{S M}}{\Gamma_{l}^{S M}} \\
& =\frac{2\left(\Delta_{1} \Gamma_{d R}^{S M}+\Delta_{2} \Gamma_{s R}^{S M}+\Delta_{3} \Gamma_{b R}^{S M}\right)}{\Gamma_{l}^{S M}}
\end{aligned}
$$

where $\Gamma_{q R}^{S M}, q=d, s, b$ denote the widths of $Z$ boson decaying into only right-handed $q$ quarks in the SM. The other is $R_{b} \equiv \Gamma_{b} / \Gamma_{h}$, where $\Gamma_{b}$ is the width into $b \bar{b}$. Explicitly, we can write $R_{b}$ as

$$
\begin{aligned}
R_{b} & =\frac{\Gamma_{b}}{\Gamma_{h}} \\
& =\frac{1+2 \Delta_{3} \frac{\Gamma_{b R}^{S M}}{\Gamma_{b}^{S M}}}{1+2 \Delta_{1} \frac{\Gamma_{d R}^{S M}}{\Gamma_{h}^{S M}}+2 \Delta_{2} \frac{\Gamma_{s R}^{S M}}{\Gamma_{h}^{S M}}+2 \Delta_{3} \frac{\Gamma_{b R}^{S M}}{\Gamma_{h}^{S M}}} \frac{\Gamma_{b}^{S M}}{\Gamma_{h}^{S M}} .
\end{aligned}
$$

Thus, the correction to $R_{b}$ is given by

$$
\delta R_{b} \approx 2\left[\Delta_{3} \frac{\Gamma_{b R}^{S M}}{\Gamma_{b}^{S M}}\left(1-\frac{\Gamma_{b}^{S M}}{\Gamma_{h}^{S M}}\right)-\Delta_{1} \frac{\Gamma_{d R}^{S M}}{\Gamma_{d}^{S M}} \frac{\Gamma_{d}^{S M}}{\Gamma_{h}^{S M}}-\Delta_{2} \frac{\Gamma_{s R}^{S M}}{\Gamma_{s}^{S M}} \frac{\Gamma_{s}^{S M}}{\Gamma_{h}^{S M}}\right] R_{b}^{S M} .
$$

The experiments give $R_{e}=20.804 \pm 0.050, R_{\mu}=20.785 \pm 0.033, R_{\tau}=20.764 \pm 0.045$ and $R_{b}=0.2163 \pm 0.0007$, respectively, while the SM predictions are $R_{e}^{S M}=R_{\mu}^{S M}=20.735$, 


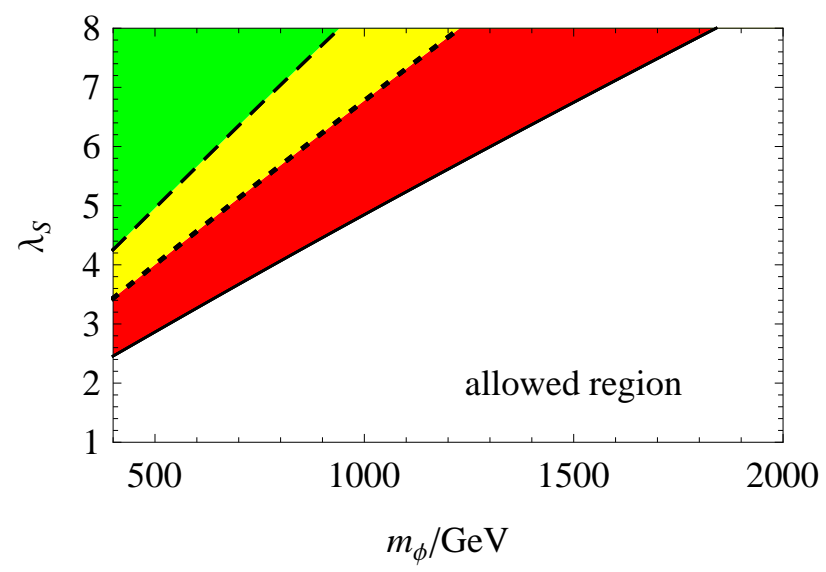

FIG. 2: The allowed region by $Z$ boson hadronic decay branching fraction as a function of $m_{\phi}$. The solid and dashed lines are the upper limits given by $R_{\tau}$ for the cases $\lambda_{S}^{12}=\lambda_{S}^{13}=\lambda_{S}^{23}=\lambda_{S}$ and $\lambda_{S}^{12}=\lambda_{S}, \lambda_{S}^{13}=\lambda_{S}^{23}=0$, respectively. The dotted line is the upper limit given by $R_{b}$ for $\lambda_{S}^{12}=\lambda_{S}, \lambda_{S}^{13}=\lambda_{S}^{23}=0$.

$R_{\tau}^{S M}=20.780$ and $R_{b}^{S M}=0.2158$ [20]. The requirement that the corrected $R_{e, \mu, \tau, b}$ are in the $1 \sigma$ range around the experimental central values imposes constraints as follows,

$$
\left(\lambda_{1}^{2}+\lambda_{2}^{2}+\lambda_{3}^{2}\right) \frac{g(a)}{4 \pi^{2}}<0.829,0.578,0.202 \text { for } R_{e}, R_{\mu}, R_{\tau}, \text { respectively }
$$

and

$$
-0.0289<\left[0.78 \lambda_{3}^{2}-0.22\left(\lambda_{1}^{2}+\lambda_{2}^{2}\right)\right] \frac{g(a)}{4 \pi^{2}}<0.173 \text { for } R_{b}
$$

We show the allowed region by $R_{\tau}$ and $R_{b}$ for $\lambda_{S}$ as a function of $m_{\phi}$ in Fig. 2. The solid and dashed lines are the upper limits given by $R_{\tau}$ for the cases $\lambda_{S}^{12}=\lambda_{S}^{13}=\lambda_{S}^{23}=\lambda_{S}$ and $\lambda_{S}^{12}=\lambda_{S}, \lambda_{S}^{13}=\lambda_{S}^{23}=0$, respectively. The dotted line is the upper limit given by $R_{b}$ for $\lambda_{S}^{12}=\lambda_{S}, \lambda_{S}^{13}=\lambda_{S}^{23}=0$. From Fig. 2 we can see that this constraint on the parameter is not very stringent. This is due to the fact that only right-handed couplings are corrected, and the widths of $Z$ boson decaying into right-handed quarks are much less than into left-handed quarks.

\section{B. $K^{0}-\overline{K^{0}}$ mixing}

Now we consider the constraint from $K^{0}-\overline{K^{0}}$ mixing. The typical Feynman diagram for $K^{0}-\overline{K^{0}}$ mixing is shown in Fig. 3. After straightforward calculations, we can obtain 


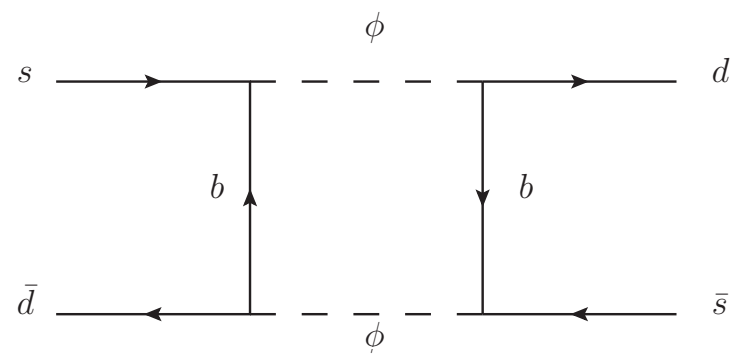

FIG. 3: Representative Feynman diagram for $K^{0}-\overline{K^{0}}$ mixing.

$$
\mathcal{H}_{\text {eff }}^{\Delta S=2}=C Q
$$

where $Q$ is the operator $\bar{d}_{R}^{\alpha} \gamma^{\mu} s_{R}^{\alpha} \bar{d}_{R}^{\beta} \gamma^{\mu} s_{R}^{\beta}$, and $C$ is its Wilson coefficient,

$$
C=\frac{\left(\lambda_{S}^{13}-\lambda_{S}^{31}\right)^{2}\left(\lambda_{S}^{23}-\lambda_{S}^{32}\right)^{2}}{32 \pi^{2}}\left[\frac{m_{\phi}^{4}-m_{b}^{4}-2 m_{b}^{2} m_{\phi}^{2} \ln \frac{m_{\phi}^{2}}{m_{b}^{2}}}{\left(m_{\phi}^{2}-m_{b}^{2}\right)^{3}}\right] b(\mu)
$$

where

$$
b(\mu)=\left(\alpha_{s}(\mu)\right)^{-2 / 9}\left(1+\frac{307}{162} \frac{\alpha_{s}(\mu)}{4 \pi}\right)
$$

contains the renormalization scale dependence [21]. We have compared this result with that in Refs. [22, 23] and find our result is consistent with their results. Then, the $K_{L}-K_{S}$ mass difference $\Delta m_{K}$ is given by 24]

$$
\Delta m_{K}=2 \operatorname{Re}\left\langle K^{0}\left|\mathcal{H}_{e f f}^{\Delta S=2}\right| \overline{K^{0}}\right\rangle=2 C \operatorname{Re}\left\langle K^{0}|Q| \overline{K^{0}}\right\rangle .
$$

The matrix element $\left\langle K^{0}|Q| \overline{K^{0}}\right\rangle$ can be parameterized as

$$
\left\langle K^{0}|Q| \overline{K^{0}}\right\rangle=\frac{1}{3} m_{K} f_{K}^{2} B_{K}(\mu)
$$

where $m_{K}$ is the mass of $K^{0}(497.6 \mathrm{MeV}), f_{K}$ is kaon decay constant $(160 \mathrm{MeV})$, and $B_{K}(\mu)$ is related to the renormalization group invariant parameter $\hat{B}_{K}$ by

$$
\hat{B}_{K}=B_{K}(\mu) b(\mu)
$$

In our numerical analysis we will use the following result [25]:

$$
\hat{B}_{K}=0.75 \pm 0.15
$$

On the other hand, the SM contribution to $\Delta m_{K}$ is

$$
\Delta m_{K}^{S M}=\frac{G_{F}^{2}}{6 \pi^{2}} f_{K}^{2} \hat{B}_{K} m_{K} M_{W}^{2} \operatorname{Re}\left[\lambda_{c}^{* 2} \eta_{1} S_{0}\left(x_{c}\right)+\lambda_{t}^{* 2} \eta_{2} S_{0}\left(x_{t}\right)+2 \lambda_{c}^{*} \lambda_{t}^{*} \eta_{3} S_{0}\left(x_{c}, x_{t}\right)\right]
$$




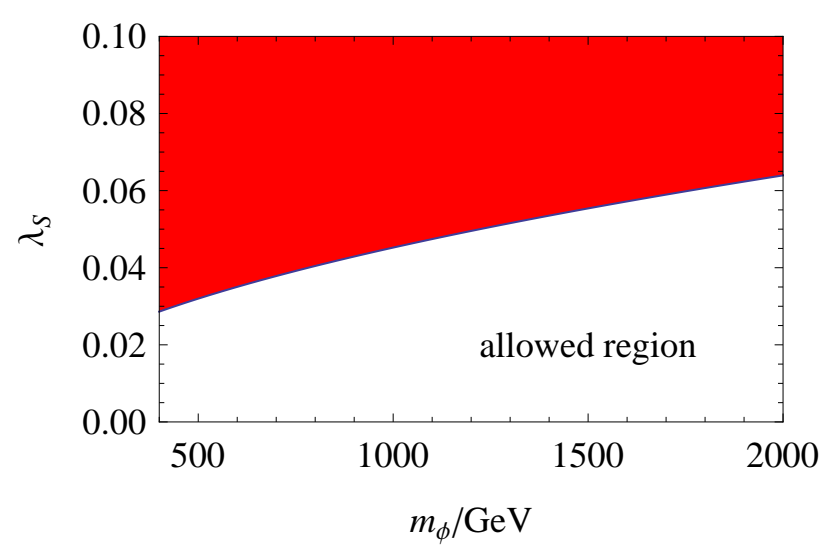

FIG. 4: The allowed region of $\lambda_{S}\left(=\lambda_{S}^{13}=\lambda_{S}^{23}\right)$ by $K^{0}-\overline{K^{0}}$ mixing as a function of $m_{\phi}$.

where $\lambda_{i}=V_{i s}^{*} V_{i s}$, and $V_{i j}$ are the CKM matrix elements. The functions $S_{0}$ are given by

$$
\begin{aligned}
S_{0}\left(x_{t}\right) & =\frac{4 x_{t}-11 x_{t}^{2}+x_{t}^{3}}{4\left(1-x_{t}\right)^{2}}-\frac{3 x_{t}^{3} \ln x_{t}}{2\left(1-x_{t}\right)^{3}}, \\
S_{0}\left(x_{c}\right) & =x_{c}, \\
S_{0}\left(x_{c}, x_{t}\right) & =x_{c}\left[\ln \frac{x_{t}}{x_{c}}-\frac{3 x_{t}}{4\left(1-x_{t}\right)}-\frac{3 x_{t}^{2} \ln x_{t}}{4\left(1-x_{t}\right)^{2}}\right]
\end{aligned}
$$

with $x_{i}=m_{i}^{2} / M_{W}^{2}$. The next-to-leading values of $\eta_{i}$ are given as follows [26 28]:

$$
\eta_{1}=1.38 \pm 0.20, \quad \eta_{2}=0.57 \pm 0.01, \quad \eta_{3}=0.47 \pm 0.04
$$

We require that the contribution to $\Delta m_{K}$, including the SM and new physics result, is not larger than the experimental value $\Delta m_{K}^{\exp }=(3.483 \pm 0.006) \times 10^{-15} \mathrm{GeV}$ 20 within $1 \sigma$ level, assuming the CPT conservation. In Fig. 4, we show the allowed region for $\lambda_{S}$ as a function of $m_{\phi}$ for $\lambda_{S}^{13}=\lambda_{S}^{23}=\lambda_{S}$. From Fig. 1 we find that the constraint on $\lambda_{S}$ is very stringent, generally less than 0.06. Furthermore, these couplings involves the third generation quarks, the parton distribution functions (PDFs) of which are small compared with those of the first two generations. Therefore, we choose $\lambda_{S}^{13}=\lambda_{S}^{23}=0$, for simplicity, in the following discussion.

\section{Dijet production at the LHC}

The dijet experiments at the LHC have set upper limits on the product of cross section $\left(\sigma_{j j}\right)$ and signal acceptance $(\mathcal{A})$ for resonance productions [29 33], such as excited quarks, axigluons, Randall-Sundrum gravitons, diquarks and string resonances. We can use these 


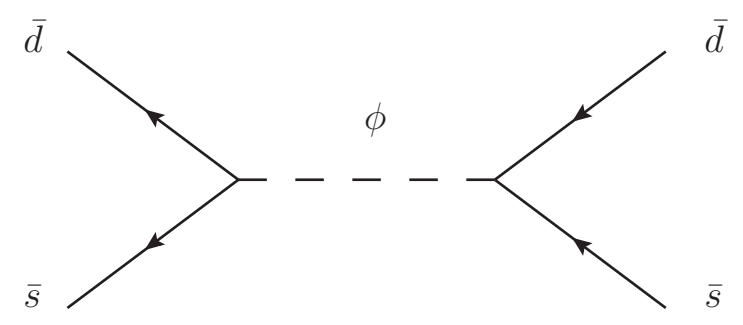

FIG. 5: Feynman diagram for the dijet production.

data to constrain the parameters in the effective Lagrange in Eq. (1). The relevant Feynman diagram for the dijet production is shown in Fig. 5.

The cross section of the resonance $\phi$ production and decaying into dijet is highly sensitive to the width of $\phi$ decay, which is given by

$$
\Gamma_{\phi}=\Gamma_{\phi \rightarrow \bar{d} \bar{s}}+\Gamma_{\phi \rightarrow u_{i} \bar{\chi}}
$$

with

$$
\begin{aligned}
\Gamma_{\phi \rightarrow \bar{d} \bar{s}} & =\frac{\left(\lambda_{S}^{12}\right)^{2}}{2 \pi} m_{\phi} \\
\Gamma_{\phi \rightarrow u_{i} \bar{\chi}} & =\frac{\left|a_{S}^{i}\right|^{2}}{16 \pi m_{\phi}^{3}}\left(m_{\phi}^{2}-m_{u_{i}}^{2}-m_{\chi}^{2}\right) \lambda^{1 / 2}\left(m_{\phi}^{2}, m_{u_{i}}^{2}, m_{\chi}^{2}\right)
\end{aligned}
$$

where $\lambda(a, b, c)=a^{2}+b^{2}+c^{2}-2 a b-2 b c-2 c a$. We will take into account the effect of these widths in our numerical calculation below. To calculate the cross section, we use MadGraph5v1.3.3 [34] with the effective Lagrangian implemented in by FeynRules [35]. We vary the mass of $\phi$ from $500 \mathrm{GeV}$ to $2500 \mathrm{GeV}$ with a step of $100 \mathrm{GeV}$. For each mass, we calculate the decay width of $\phi$, assuming $\lambda_{S}^{12}=a_{S}^{3}=0.2, a_{S}^{1}=a_{S}^{2}=0, m_{\chi}=50 \mathrm{GeV}$. Then we change the corresponding parameters in MadGraph and calculate the cross sections of the dijet production. We choose the kinematical cuts as following [29, 32]:

$$
\left|\eta_{j}\right|<2.5, \quad\left|\eta_{j_{1}}-\eta_{j_{2}}\right|<1.3
$$

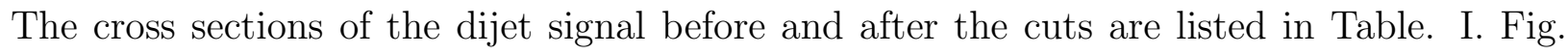
6 shows the allowed region of $\lambda_{S}\left(=\lambda_{S}^{12}=a_{S}^{3}\right)$ as a function of $m_{\phi}$, where we choose the acceptance $\mathcal{A}=0.6$ as in Ref. [32]. It is required that $\sigma_{j j} \cdot \mathcal{A}$ is not larger than the observed 95\% C.L. upper limit in the dijet experiment [29, 32]. The bump of the curve in the region from $500 \mathrm{GeV}$ to $1000 \mathrm{GeV}$ for $m_{\phi}$ is due to the fact that we compare with data in this 


\begin{tabular}{lccccccc}
\hline \hline$m_{\phi}(\mathrm{GeV})$ & 500 & 600 & 700 & 800 & 900 & 1000 & 1100 \\
\hline \hline$\sigma_{0}(\mathrm{pb})$ & 28.2 & 12.6 & 6.11 & 3.17 & 1.73 & $9.80 \times 10^{-2}$ & $5.71 \times 10^{-2}$ \\
$\sigma_{f}(\mathrm{pb})$ & 16.2 & 7.13 & 3.52 & 1.84 & $9.98 \times 10^{-2}$ & $5.66 \times 10^{-2}$ & $3.22 \times 10^{-2}$ \\
\hline \hline$m_{\phi}(\mathrm{GeV})$ & 1200 & 1300 & 1400 & 1500 & 1600 & 1700 & 1800 \\
\hline \hline$\sigma_{0}(\mathrm{pb})$ & $3.40 \times 10^{-2}$ & $2.07 \times 10^{-2}$ & $1.28 \times 10^{-2}$ & $7.95 \times 10^{-2}$ & $5.00 \times 10^{-2}$ & $3.17 \times 10^{-2}$ & $2.03 \times 10^{-2}$ \\
$\sigma_{f}(\mathrm{pb})$ & $1.94 \times 10^{-2}$ & $1.18 \times 10^{-2}$ & $7.27 \times 10^{-2}$ & $4.55 \times 10^{-2}$ & $2.82 \times 10^{-2}$ & $1.79 \times 10^{-2}$ & $1.16 \times 10^{-2}$ \\
\hline \hline$m_{\phi}(\mathrm{GeV})$ & 1900 & 2000 & 2100 & 2200 & 2300 & 2400 & 2500 \\
\hline \hline$\sigma_{0}(\mathrm{pb})$ & $1.30 \times 10^{-2}$ & $8.35 \times 10^{-3}$ & $5.38 \times 10^{-3}$ & $3.47 \times 10^{-3}$ & $2.23 \times 10^{-3}$ & $1.44 \times 10^{-3}$ & $9.27 \times 10^{-4}$ \\
$\sigma_{f}(\mathrm{pb})$ & $7.42 \times 10^{-3}$ & $4.79 \times 10^{-3}$ & $3.07 \times 10^{-3}$ & $1.99 \times 10^{-3}$ & $1.28 \times 10^{-3}$ & $8.35 \times 10^{-4}$ & $5.25 \times 10^{-4}$ \\
\hline
\end{tabular}

TABLE I: The cross sections of dijet production induced by the resonance of $\phi$ before $\left(\sigma_{0}\right)$ and after $\left(\sigma_{f}\right)$ the cuts given in Eq. (27), assuming $\lambda_{S}^{12}=a_{S}^{3}=0.2, a_{S}^{1}=a_{S}^{2}=0$.

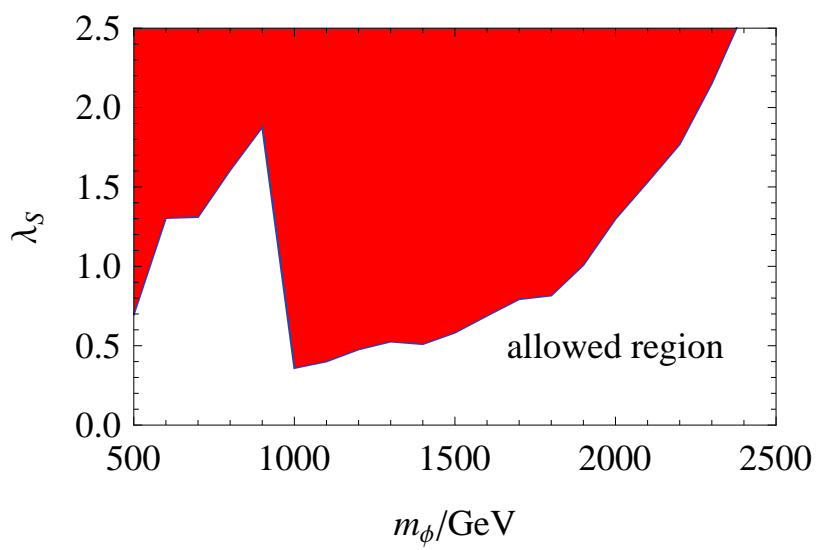

FIG. 6: The allowed region of $\lambda_{S}\left(=\lambda_{S}^{12}=a_{S}^{3}\right)$ by dijet experiments at the LHC as a function of $m_{\phi}$.

region and the other regions corresponding to integrated luminosities of $2.9 \mathrm{pb}^{-1}$ and $1 \mathrm{fb}^{-1}$, respectively, collected by the CMS experiment at the LHC.

\section{SIGNAL AND BACKGROUND}

The signals of the monotop production are

$$
p p \rightarrow t+E_{T} \rightarrow b W+E_{T} \rightarrow b j j+E_{T} \text { and } \quad b l+E_{T},
$$



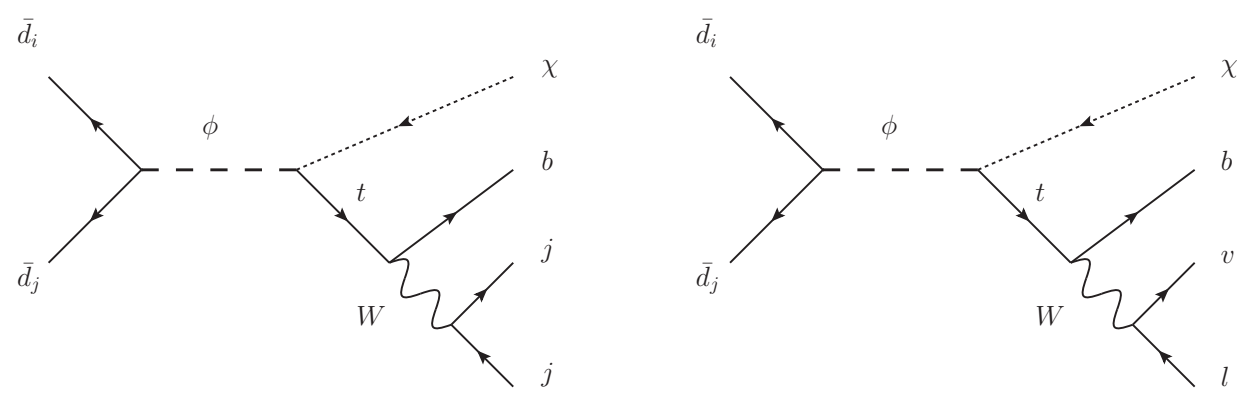

FIG. 7: Feynman diagrams for the monotop production.

which are shown in Fig. 7. The symbol $b$ and $j$ denote a $b$-tagged jet and light quark or gluon jet, respectively, and $l$ refers to the first two generation charged leptons, i.e., $e$ and $\mu$. We define the process with top hadronic decay as hadronic mode, while the one with top semileptonic decay as semileptonic mode. The hadronic mode suffers from fewer backgrounds in the SM than the semileptonic mode because of the smaller phase space due to more particles in the final states. This mode has been studied in Ref. [11] where they assume the branching fraction $R(\phi \rightarrow t \bar{\chi})$ equal to one. However, this assumption is over optimistic. From Eq. (26) we get the branching fraction $R(\phi \rightarrow t \bar{\chi})$,

$$
R(\phi \rightarrow t \bar{\chi})=\frac{\Gamma_{\phi \rightarrow t \bar{\chi}}}{\Gamma_{\phi \rightarrow t \bar{\chi}}+\Gamma_{\phi \rightarrow \bar{d} \bar{s}}}=\frac{1}{1+z},
$$

with

$$
z=\frac{8\left(\lambda_{S}^{12}\right)^{2}}{\left|a_{S}^{3}\right|^{2}} \frac{m_{\phi}^{4}}{\left(m_{\phi}^{2}-m_{t}^{2}-m_{\chi}^{2}\right) \lambda^{1 / 2}\left(m_{\phi}^{2}, m_{t}^{2}, m_{\chi}^{2}\right)} .
$$

Here we assume that the decay widths $\Gamma_{\phi \rightarrow u \bar{\chi}}=\Gamma_{\phi \rightarrow c \bar{\chi}}=0$. In the case of $\lambda_{S}^{12}=a_{S}^{3}=$ $0.2, m_{t}=173.1 \mathrm{GeV}, m_{\phi}=500 \mathrm{GeV}$ and $m_{\chi}=50 \mathrm{GeV}$, we find $\Gamma_{\phi \rightarrow t \bar{\chi}}=0.300 \mathrm{GeV}$, $\Gamma_{\phi \rightarrow \bar{d} \bar{s}}=3.183 \mathrm{GeV}$, and the branching fraction of $\phi \rightarrow t \bar{\chi}$ is just about 0.1. So, in this work, we take into account the effect of both $\phi$ decay channels and below we will discuss further the hadronic and leptonic modes in detail.

Before discussing the signal and backgrounds in detail, we first give some comments on the parameter $m_{\chi}$. In the SUSY model, without the assumption of gaugino mass unification, there is no general mass limit from $e^{+} e^{-}$colliders for the lightest neutralino [20]. The indirect constraints from $(g-2)_{\mu}, b \rightarrow s \gamma$ and $B \rightarrow \mu^{+} \mu^{-}$show that the lightest neutralino mass can be as low as about $6 \mathrm{GeV}$ [36]. In our case, we choose the default value of $m_{\chi}=50 \mathrm{GeV}$ and discuss the effect on the discovery significance when varying $m_{\chi}$ in the range $5-100$ 
GeV. An estimate of the width can be made by the Feynman diagrams shown in Fig. 0, where we can consider only $\chi$ as the initial-state particle, for example,

$$
\chi\left(p_{1}\right) \rightarrow d\left(p_{2}\right) s\left(p_{3}\right) b\left(p_{4}\right) \nu\left(p_{5}\right) l^{+}\left(p_{6}\right) .
$$

Then the width of $\chi$ is given by

$$
\Gamma_{\chi}=\frac{1}{2 m_{\chi}} \int|\overline{\mathcal{M}}|^{2} d \Gamma_{5}
$$

where $|\overline{\mathcal{M}}|^{2}$ is the matrix element squared for the decay process which has taken into account the average and sum over the initial- and final-state spins and colors. When the masses of all the final-state particles are neglected, the five body phase space integration can be written as

$$
\begin{aligned}
\int d \Gamma_{5}= & \frac{1}{32768 \pi^{7}} \frac{1}{m_{\chi}^{2}} \int_{0}^{m_{\chi}^{2}} d s_{23} \int_{0}^{\left(m_{\chi}-\sqrt{s_{23}}\right)^{2}} d s_{456} \int_{0}^{s_{456}} d s_{45} \\
& \lambda^{1 / 2}\left(m_{\chi}^{2}, s_{23}, s_{456}\right)\left(1-\frac{s_{45}}{s_{456}}\right)
\end{aligned}
$$

where $s_{i j}=\left(p_{i}+p_{j}\right)^{2}$ and $s_{i j k}=\left(p_{i}+p_{j}+p_{k}\right)^{2}$. In the mass range of $\chi$ we are interested in, the momenta of the decay products of the $W$ boson are so small compared with the mass of the $W$ boson that we neglect them in the calculation of the matrix element. Moreover, we assume that the lepton $l^{+}$carries about one-fifth of the energy of $\chi$ on average. In this case, the matrix element squared is simply given by

$$
|\overline{\mathcal{M}}|^{2} \approx \frac{96}{5} g_{W}^{4}\left(\lambda_{S}^{12}\right)^{2}\left(a_{S}^{3}\right)^{2} \frac{m_{\chi}^{2}}{m_{\phi}^{4} m_{t}^{2} M_{W}^{4}} s_{23} s_{45}
$$

where $g_{W}$ is the coupling of the $W$ boson with left-handed fermions. Then we perform the integration in Eq. (32), and obtain

$$
\Gamma_{\chi} \approx 1.82 \times 10^{-19} \mathrm{GeV}\left(\frac{\lambda_{S}^{12}}{0.2}\right)^{2}\left(\frac{a_{S}^{3}}{0.2}\right)^{2} \frac{\left(m_{\chi} / 50 \mathrm{GeV}\right)^{11}}{\left(m_{\phi} / 500 \mathrm{GeV}\right)^{4}\left(m_{t} / 173.1 \mathrm{GeV}\right)^{2}\left(M_{W} / 80.4 \mathrm{GeV}\right)^{4}}
$$

The produced $\chi$ at hadron colliders, as a decay product of a massive particle, usually has such a large energy that it moves nearly in the speed of light. In Fig. 8, we show the distance travelled by the particle $\chi$ before its decay as a function of its mass. It can be seen that the distance strongly depends on the mass of $\chi$ and decreases with increasing $m_{\chi}$. The results of MadGraph are well approximated by those obtained from Eq. (35) except 


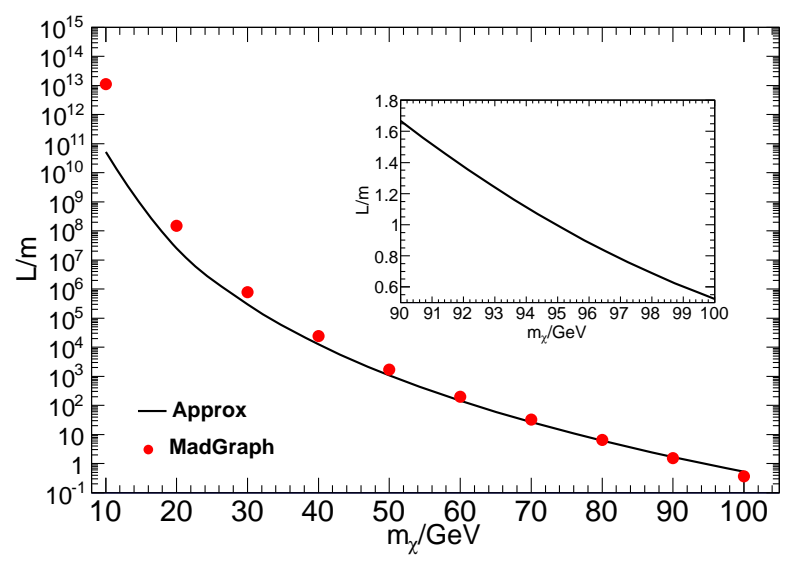

FIG. 8: The distance travelled by the particle $\chi$ before its decay as a function of its mass. The solid line is obtained from Eq. (35) while the dots denote the results of MadGraph. The relevant parameters are chosen as $\lambda_{S}^{12}=a_{S}^{3}=0.2, m_{t}=173.1 \mathrm{GeV}, M_{W}=80.4 \mathrm{GeV}$, and $m_{\phi}=500 \mathrm{GeV}$.

for the low mass region since we have neglected the mass of final-state particle in Eq. (35). But this discrepancy between them in the low mass region is not important because they are both much larger than the size of the detector at the LHC. The ATLAS collaboration has searched for displaced vertices arising from decays of new heavy particles and found that the efficiency for detecting displaced vertices almost vanishes for a distance between the primary and the displaced vertex larger than $0.35 \mathrm{~m}$ [37]. Therefore, as shown in Fig. 8, it is reasonable that the particle $\chi$ with a mass less than $100 \mathrm{GeV}$ is considered as missing energy at the LHC.

\section{A. Hadronic mode}

For the hadronic mode, the main backgrounds arise from $p p \rightarrow j j j Z(\nu \bar{\nu})$, with a jet misidentified as a $b$-jet, and $p p \rightarrow b \bar{b} j Z(\nu \bar{\nu})$ with a $b$-jet not tagged. The top pair and single top production processes with hadronic top quark decay may also contribute to the backgrounds if some jets are not detected. The signal and backgrounds are simulated by MadGraph5v1.3.3 [34] and ALPGEN [38] interfaced with PYTHIA [39, 40] to perform the parton shower and hadronization. In this mode, the momentum of three jets, and therefore momentum of the $W$ boson and top quark, can be reconstructed, which leads to efficient event selection using invariant mass cut. In the following numerical calculation, the default 
relevant parameters are chosen as $\lambda_{S}^{12}=a_{S}^{3}=0.2, \lambda_{S}^{13}=\lambda_{S}^{23}=0, a_{S}^{1}=a_{S}^{2}=0, m_{t}=$ $173.1 \mathrm{GeV}, m_{\phi}=500 \mathrm{GeV}$ and $m_{\chi}=50 \mathrm{GeV}$, and CTEQ6L1 PDF is used. The renormalization and factorization scales are set at $m_{\phi}$. We use the following basic selection cuts

$$
p_{T}^{b, j}>30 \mathrm{GeV}, \quad\left|\eta^{b, j}\right|<2.4, \quad \Delta R_{b b, b j, j j}>0.5 .
$$

Moreover, we choose a $b$-tagging efficiency of $50 \%$ while the misidentification rates for other jets are, $8 \%$ for charm quark, $0.2 \%$ for gluon and other light quarks [11.

To determine the missing transverse energy cut, we show the normalized spectrum of the missing transverse energy for the signal and backgrounds in Fig. 9. The backgrounds concentrate in the region $E_{T}<100 \mathrm{GeV}$ because the missing transverse energy of the background comes from either an invisible decayed $Z$ boson or non-detected jets, which are produced mainly via t-channel. In contrast, the missing transverse energy of the signal results from the decay of a heavy resonance so that it can be large. Therefore we choose the missing transverse energy cut

$$
E_{T}>100 \mathrm{GeV} \text {. }
$$

Meanwhile, the shape of the signal is similar to the distribution $E_{T} / \sqrt{E_{T}^{\max 2}-E_{T}^{2}}$ with an edge at $E_{T}^{\max }=\lambda^{1 / 2}\left(m_{\phi}^{2}, m_{t}^{2}, m_{\chi}^{2}\right) / 2 m_{\phi}$. This feature may help to specify the masses of the resonance and the missing particle.

In Fig. 10 we show the reconstructed top quark mass distribution for the signal and backgrounds processes using the three leading jets. It can be seen that there is a peak around $175 \mathrm{GeV}$ for the signal while the distributions of backgrounds grow up with the increase of reconstructed top quark mass, and thus we impose the invariant mass cut in the final states as following,

$$
120 \mathrm{GeV}<m_{t, r}<200 \mathrm{GeV} .
$$

The cross sections of the signal and backgrounds after various cuts at the LHC $(\sqrt{s}=7$ $\mathrm{TeV}$ ) are listed in Table [1]. It can be seen that the backgrounds decrease dramatically when the invariant mass cuts are imposed, and the cross section of $b \bar{b} j Z(\nu \bar{\nu})$ is not smaller than that of $j j j Z(\nu \bar{\nu})$ after all cuts imposed so that it can not be neglected. The $t \bar{t}$ and $t(\bar{t}) j$ processes are mainly suppressed by the missing transverse energy cut, which can be seen from Fig. 9 . 


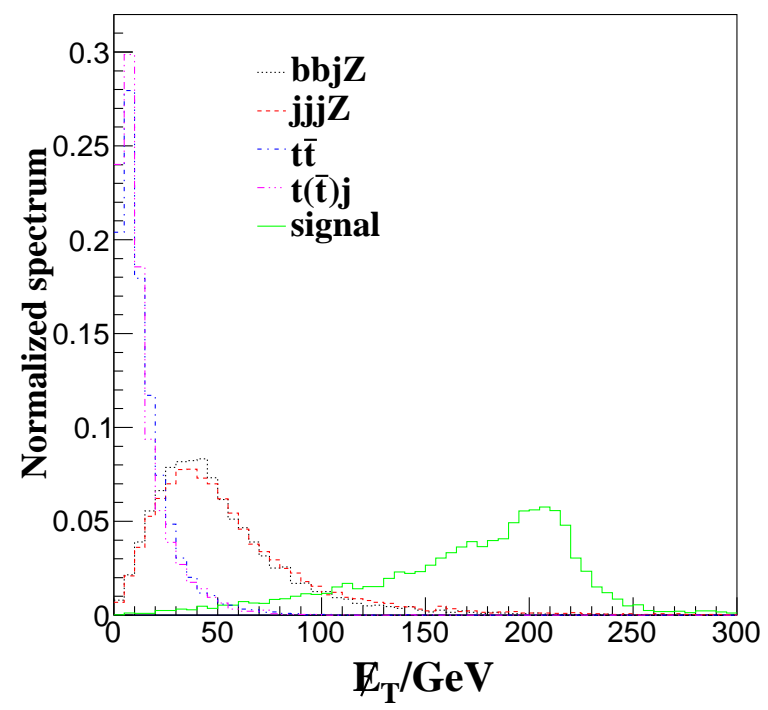

FIG. 9: The normalized spectrum of missing transverse energy in the hadronic mode at the LHC $(\sqrt{s}=7 \mathrm{TeV})$.

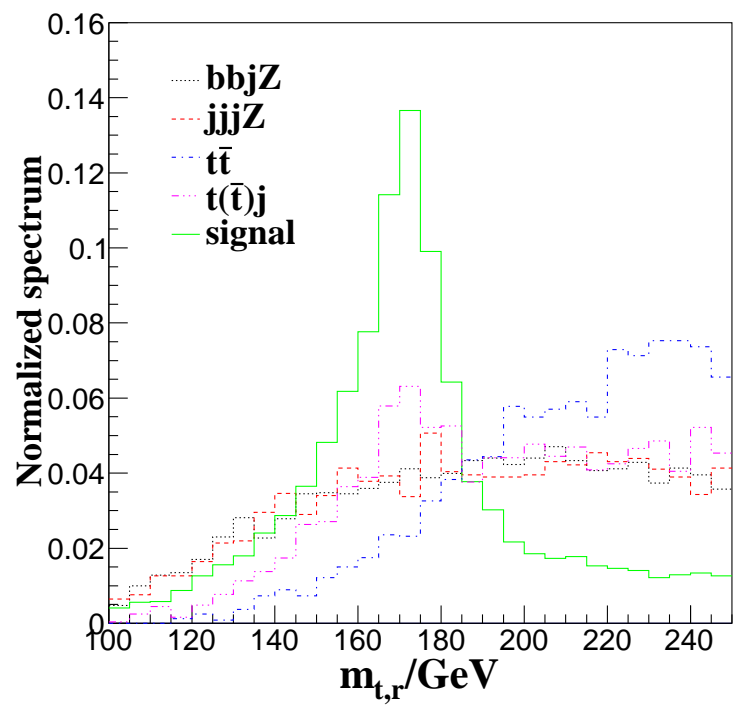

FIG. 10: The reconstructed top quark mass distribution for the signal and backgrounds processes.

To investigate the discovery potential of monotops in the hadronic mode at the LHC $(\sqrt{s}=7 \mathrm{TeV})$ with an integrated luminosity of $1 \mathrm{fb}^{-1}$, in Fig. 11 we present the contour curves of significance $\mathcal{S}=S / \sqrt{B}$ versus the parameters $\lambda_{S}^{12}$ and $a_{S}^{3}$, where $S$ and $B$ are respectively the expected numbers of the signal and backgrounds events. And in Fig. 12 


\begin{tabular}{|c|c|c|c|c|c|}
\hline$\sigma(\mathrm{fb})$ & basic & $E_{T}$ & $m_{t, r}$ & b-tagging & $\epsilon_{\text {cut }}$ \\
\hline signal & 902 & 811 & 502 & 251 & $27.1 \%$ \\
\hline$j j j Z(\nu \bar{\nu})$ & $7.03 \times 10^{4}$ & $7.87 \times 10^{3}$ & 944 & 9.35 & $0.013 \%$ \\
\hline$b \bar{b} j Z(\nu \bar{\nu})$ & $1.70 \times 10^{3}$ & 143 & 19.4 & 9.67 & $0.57 \%$ \\
\hline$t \bar{t}$ & $2.80 \times 10^{4}$ & 34.6 & 0.28 & 0.14 & $5 \times 10^{-6}$ \\
\hline$t(\bar{t}) j$ & $2.35 \times 10^{4}$ & 10.9 & 0.24 & 0.12 & $5 \times 10^{-6}$ \\
\hline
\end{tabular}

TABLE II: The cross sections of the signal and backgrounds after various cuts in the hadronic mode at the $\operatorname{LHC}(\sqrt{s}=7 \mathrm{TeV})$. The cut acceptance $\epsilon_{c u t}$ is also listed. The entries after the $m_{t, r}$ cut for $t \bar{t}$ and $t(\bar{t}) j$ processes are estimated by considering that one out of the total events we have generated for analysis can survive various kinematic cuts.

we present the $5 \sigma(\mathcal{S}=5)$ discovery limits of $m_{\phi}, m_{\chi}$ and $\lambda_{S}^{12}=a_{S}^{3}=\lambda_{S}$. From Fig. 11 we can see that for a $5 \sigma$ discovery, the sensitivity to $\lambda_{S}^{12}$ and $a_{S}^{3}$ can be as low as 0.02 and 0.06, respectively. And from Fig. 12, we find that the LHC can generally detect the coupling $\lambda_{S}$ down to lower than 1.0 for $m_{\phi}$ less than $1.4 \mathrm{TeV}$. For $m_{\phi}$ larger than $1.4 \mathrm{TeV}$, the coupling $\lambda_{S}$ needed to discovery the monotop signal increases quickly. The increase of the integrated luminosity has a larger impact for larger $m_{\phi}$. Moreover, the narrow bands of the lines, which correspond to the value of $m_{\chi}$ varying from $5 \mathrm{GeV}$ to $100 \mathrm{GeV}$, indicate the weak dependence of the discovery potential on the value of $m_{\chi}$ if $m_{\chi} \ll m_{\phi}$.

In this mode, since the full kinematic information of the top quark can be reconstructed, the mass of the resonance $\phi$ can be obtained by

$$
m_{\phi}=\sqrt{p_{t}^{2}+m_{\chi}^{2}}+\sqrt{p_{t}^{2}+m_{t}^{2}}
$$

with

$$
p_{t}^{2}=p_{t, x}^{2}+p_{t, y}^{2}+p_{t, z}^{2}
$$

in which $p_{t, x}, p_{t, y}, p_{t, z}$ are the three-vector momentum of the top quark. Fig. 13 shows the distribution of the reconstructed $m_{\phi}$. We can see a peak around $m_{\phi}=500 \mathrm{GeV}$ in the signal. To illustrate the effect of $m_{\chi}$, we also plot the situation that $m_{\chi}=0 \mathrm{GeV}$ is assumed in Eq. (39) when reconstructing $m_{\phi}$. It is evident that the peak position does not changed. This information, combined with the missing transverse energy distribution, may help to specify the masses of the resonance and the missing particle. 


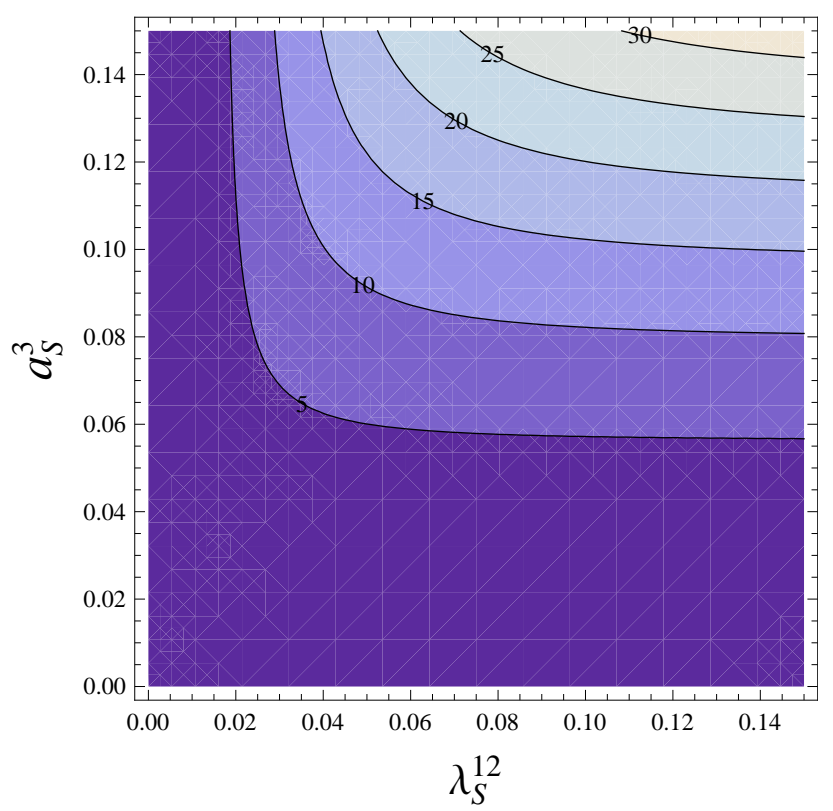

FIG. 11: The significance in the hadronic mode at the LHC $(\sqrt{s}=7 \mathrm{TeV})$ with an integrated luminosity of $1 \mathrm{fb}^{-1}$ versus the parameters $\lambda_{S}^{12}$ and $a_{S}^{3}$, assuming $m_{\phi}=500 \mathrm{GeV}$ and $m_{\chi}=50 \mathrm{GeV}$.

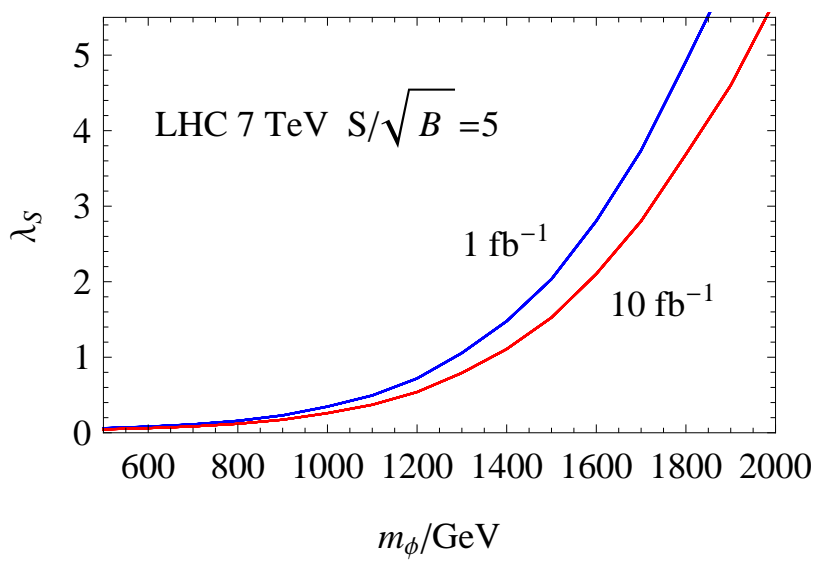

FIG. 12: The $5 \sigma$ discovery limits of $m_{\phi}$ and $\lambda_{S}\left(=\lambda_{S}^{12}=a_{S}^{3}\right)$ in the hadronic mode at the LHC $(\sqrt{s}=7 \mathrm{TeV})$. Either band consists of twenty solid lines from the bottom up corresponding to the value of $m_{\chi}$ varying from $5 \mathrm{GeV}$ to $100 \mathrm{GeV}$ with a step of $5 \mathrm{GeV}$.

\section{B. Semileptonic mode}

For the semileptonic mode, the dominant backgrounds are $p p \rightarrow W(l \nu) j$ with the jet misidentified as a $b$-jet and single top production with semileptonic top quark decay. The $W j$ background is very large because there are only two final-state particles, compared with 


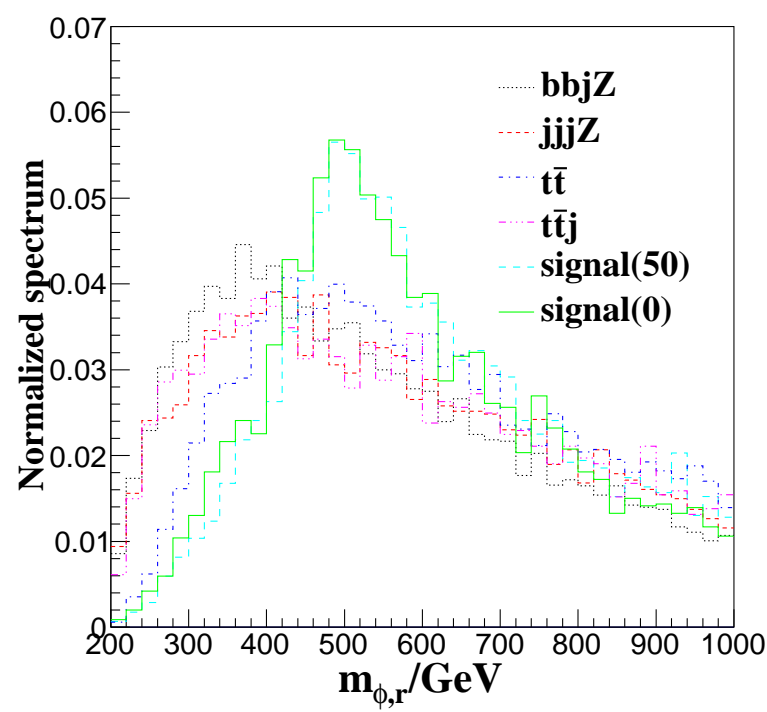

FIG. 13: The reconstructed $m_{\phi}$ distribution for the signal and backgrounds processes. The signal(50) and signal(0) represent that the values of $m_{\chi}$ in Eq. (39) are $50 \mathrm{GeV}$ and $0 \mathrm{GeV}$, respectively.

four final-state particles in $p p \rightarrow j j j Z$ and $p p \rightarrow b \bar{b} j Z$ processes. Besides, the final state of the signal contains two missing particles, which makes the reconstruction of the mass of the top quark very challenging. Nevertheless, the semileptonic mode is still promising once appropriate cuts are imposed. The signal and backgrounds are simulated by MadGraph5v1.3.3 [34] interfaced with PYTHIA [39]. We choose the same default parameters as in hadronic mode, and the basic cuts are

$$
p_{T}^{b}>30 \mathrm{GeV}, \quad\left|\eta^{b, l}\right|<2.4, \quad \Delta R_{b l}>0.5
$$

Fig. 14 shows the normalized spectrum of the transverse momentum of the charged lepton in the semileptonic mode at the LHC with $\sqrt{s}=7 \mathrm{TeV}$. We can see that it is difficult to suppress the backgrounds by $p_{T}^{l}$ cut because of the similar distributions of the signal and backgrounds. As a result, we choose a loose cut

$$
p_{T}^{l}>20 \mathrm{GeV}
$$

to keep more signal events. 


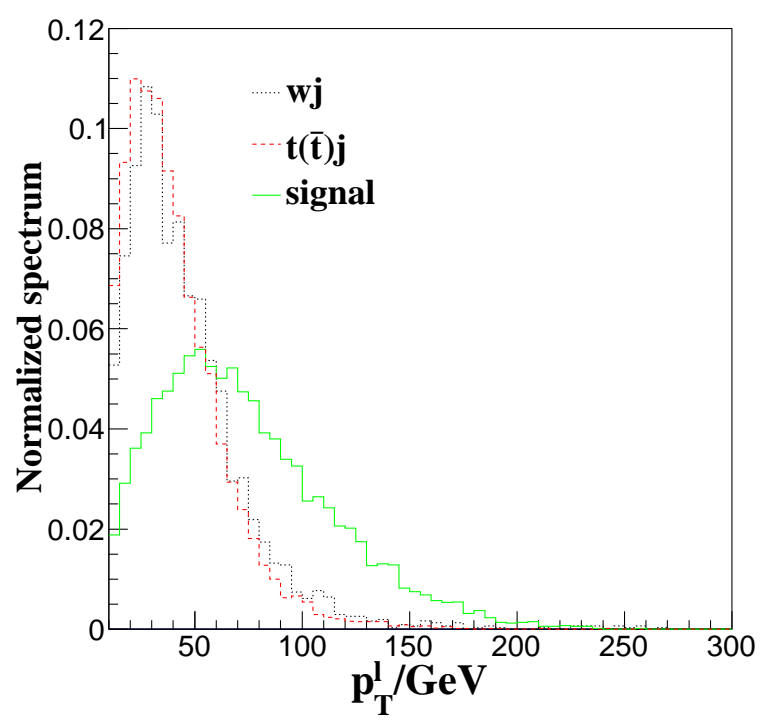

FIG. 14: The normalized spectrum of transverse momentum of the charged lepton in the semileptonic mode at the $\mathrm{LHC}(\sqrt{s}=7 \mathrm{TeV})$.

Fig. 15 shows the normalized spectrum of the missing transverse energy in the semileptonic mode at the LHC with $\sqrt{s}=7 \mathrm{TeV}$. The backgrounds decrease while the signal increases in the range $30 \mathrm{GeV}<\mathscr{E}_{T}<150 \mathrm{GeV}$. The reason is that the missing particle of the backgrounds is (anti)neutrino, which comes from the $W$ boson, and the $W j$ is mainly produced through t-channel, in which the momentum of final-state particles tend to be collinear to those of the initial-state particles. The situation for the single top production is similar. In contrast, the missing particles of the signal originate from a resonance of a large mass, and thus could be produced with large transverse momentum. Therefore, we impose the missing transverse energy cut

$$
E_{T}>120 \mathrm{GeV}
$$

to suppress the backgrounds.

Fig. 16 shows the normalized spectrum of the transverse mass, which is defined as [20]

$$
M_{T}=\sqrt{\left(E_{T}+E_{T}^{l}\right)^{2}-\left(\vec{p}_{T}+\vec{p}_{T}^{\vec{l}}\right)^{2}},
$$

in the semileptonic mode at the LHC with $\sqrt{s}=7 \mathrm{TeV}$. The backgrounds increase in the range $0<M_{T}<80 \mathrm{GeV}$ and have a peak around $M_{T} \sim 80 \mathrm{GeV}$. This is due to the fact that the transverse mass measure the maximum of the invariant mass of the missing particles and the lepton, which is the mass of $W$ boson for the backgrounds. In contrast, the signal 


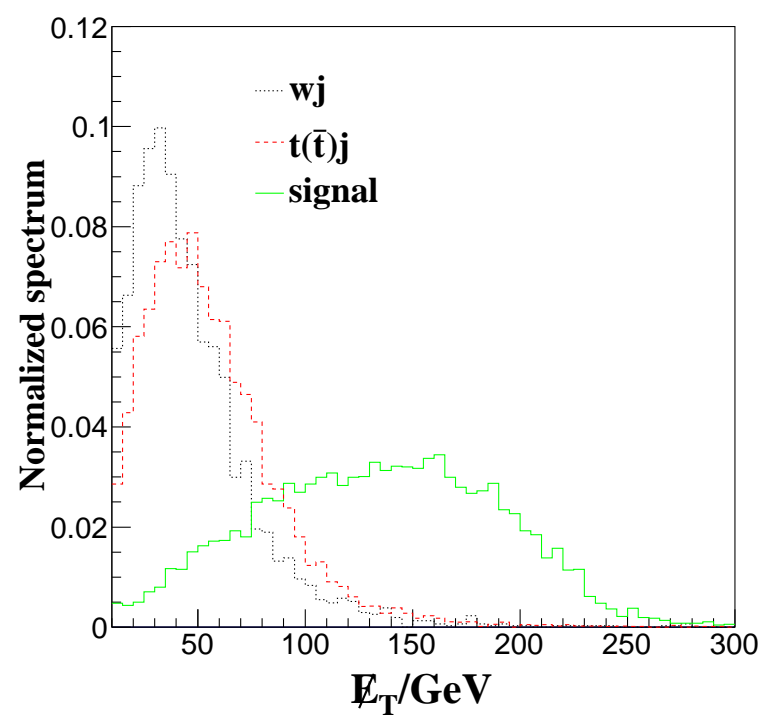

FIG. 15: The normalized spectrum of the missing transverse energy in the semileptonic mode at the $\operatorname{LHC}(\sqrt{s}=7 \mathrm{TeV})$.

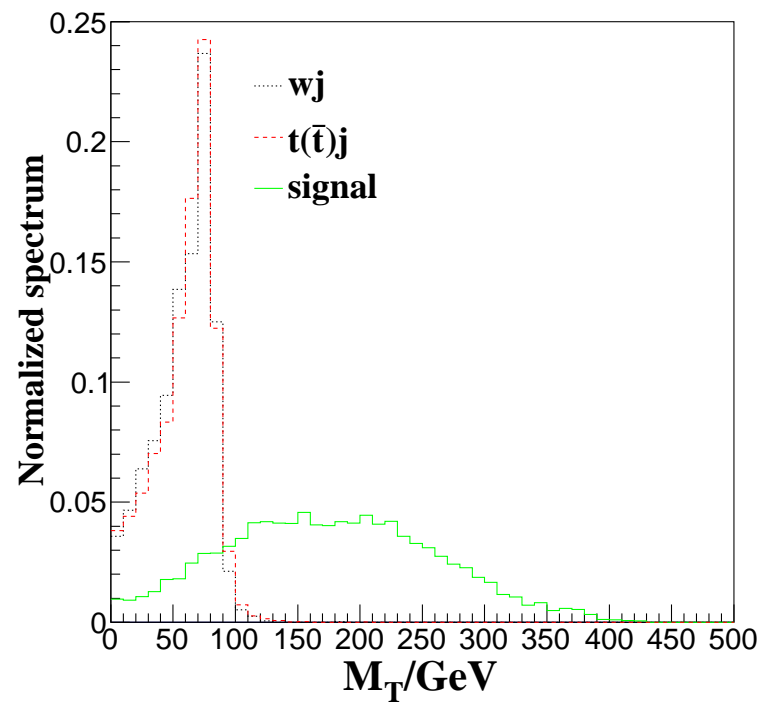

FIG. 16: The normalized spectrum of the transverse mass $M_{T}$ in the semileptonic mode at the $\operatorname{LHC}(\sqrt{s}=7 \mathrm{TeV})$.

concentrates in the range $M_{T}>100 \mathrm{GeV}$. Thus, to suppress the backgrounds efficiently, we impose the transverse mass cut

$$
M_{T}>120 \mathrm{GeV} .
$$




\begin{tabular}{lcccccc}
\hline \hline$\sigma(\mathrm{fb})$ & basic & $p_{T}^{l}$ & $E_{T}$ & $M_{T}$ & b-tagging & $\epsilon_{\text {cut }}$ \\
\hline \hline signal & 399 & 376 & 231 & 218 & 109 & $27.3 \%$ \\
$W(l \nu) j$ & $1.83 \times 10^{6}$ & $1.53 \times 10^{6}$ & $3.45 \times 10^{4}$ & 1.83 & 0.003 & $2 \times 10^{-9}$ \\
$t(\bar{t}) j$ & $9.09 \times 10^{3}$ & $7.33 \times 10^{3}$ & 185 & 2.15 & 1.08 & $0.012 \%$ \\
\hline
\end{tabular}

TABLE III: The cross sections of the signal and backgrounds after various cuts in the semileptonic mode at the $\operatorname{LHC}(\sqrt{s}=7 \mathrm{TeV})$. The cut acceptance $\epsilon_{c u t}$ is also listed. The entries after the $M_{T}$ cut for $W(l \nu) j$ process are estimated by considering that one out of the total events we have generated for analysis can survive various kinematic cuts.

The cross sections of the signal and backgrounds after various cuts at the LHC $(\sqrt{s}=$ $7 \mathrm{TeV}$ ) are listed in Table [II. We can see that the backgrounds nearly vanish after the transverse mass cut is imposed, which means that it is very promising to search for the signal of monotops in the semileptonic mode. In Fig. 17, we show the contour curves of the significance $\mathcal{S}$ versus the parameters $\lambda_{S}^{12}$ and $a_{S}^{3}$ in the semileptonic mode at the LHC $(\sqrt{s}=7 \mathrm{TeV})$. And in Fig. 18, we show the $5 \sigma(\mathcal{S}=5)$ discovery limits of $m_{\phi}, m_{\chi}$ and $\lambda_{S}^{12}=a_{S}^{3}=\lambda_{S}$ in the semileptonic mode. From Fig. 17 we can see that for a $5 \sigma$ discovery, the sensitivity to $\lambda_{S}^{12}$ and $a_{S}^{3}$ can be as low as 0.015 and 0.045 , respectively, which are smaller than the corresponding values in the hadronic mode. And from Fig. 18, we find that the LHC can generally detect the coupling $\lambda_{S}$ down to lower than 0.4 for $m_{\phi}$ less than $1.4 \mathrm{TeV}$, and for larger $m_{\phi}$, the coupling $\lambda_{S}$ needed to discover the monotop signal increases quickly. Also, the value of $m_{\chi}$ has little effect on the discovery potential.

\section{CONCLUSION}

We have investigated the potential of the early LHC to discover the signal of monotop production. First, we obtain the parameter space of the effective Lagrangian constrained by the present data of $Z$ boson hadronic decay branching ratio, $K^{0}-\overline{K^{0}}$ mixing and dijet productions at the LHC. Then, we study the various cuts imposed on the events, reconstructed from the hadronic final states, to suppress backgrounds and increase the significance in detail. And we find that in the hadronic mode the information from the missing transverse energy and reconstructed resonance mass distributions can be used to specify the masses of 


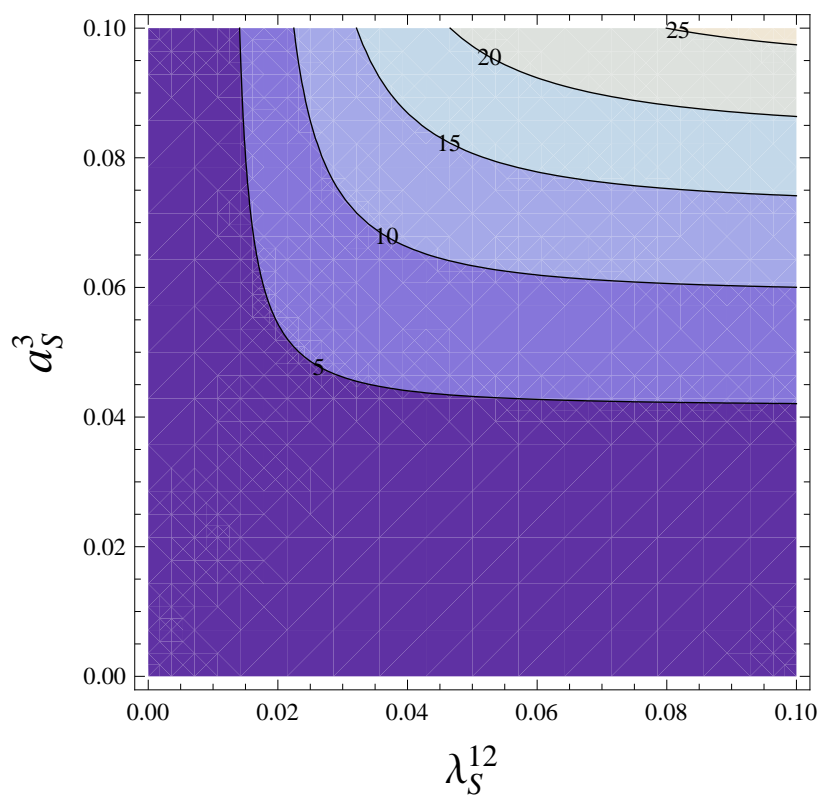

FIG. 17: The significance in the semileptonic mode at the LHC $(\sqrt{s}=7 \mathrm{TeV})$ with an integrated luminosity of $1 \mathrm{fb}^{-1}$ versus the parameters $\lambda_{S}^{12}$ and $a_{S}^{3}$, assuming $m_{\phi}=500 \mathrm{GeV}$ and $m_{\chi}=50 \mathrm{GeV}$.

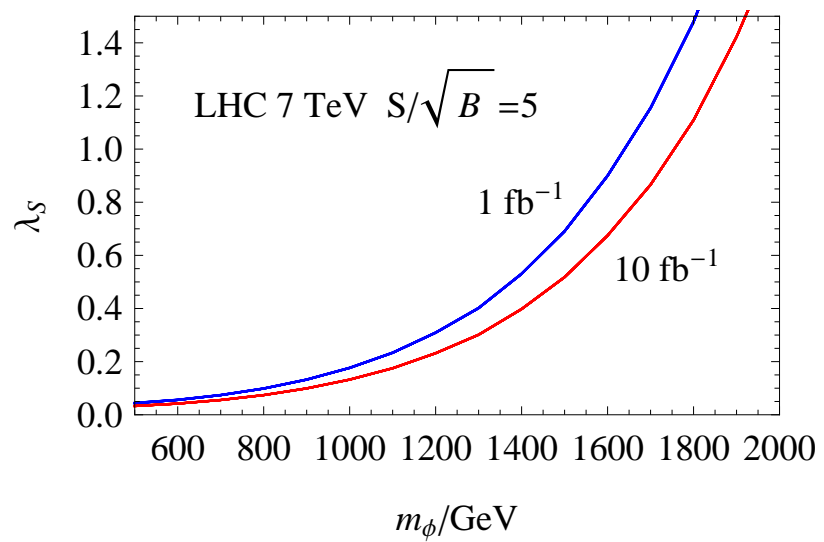

FIG. 18: The $5 \sigma$ discovery limits of $m_{\phi}$ and $\lambda_{S}\left(=\lambda_{S}^{12}=a_{S}^{3}\right)$ in the semileptonic mode at the LHC $(\sqrt{s}=7 \mathrm{TeV})$. Either band consists of twenty solid lines from the bottom up corresponding to the value of $m_{\chi}$ varying from $5 \mathrm{GeV}$ to $100 \mathrm{GeV}$ with a step of $5 \mathrm{GeV}$.

the resonance and the missing particle. Lastly, we present the significance $\mathcal{S}$ at the LHC $(\sqrt{s}=7 \mathrm{TeV})$ with an integrated luminosity of $1 \mathrm{fb}^{-1}$ in the parameter space allowed by the current data, and the $5 \sigma$ discovery limits of $m_{\phi}$ and $\lambda_{S}\left(=\lambda_{S}^{12}=a_{S}^{3}\right)$. Our results show that the LHC can generally detect the coupling $\lambda_{S}$ down to lower than 1.0 and 0.4 for $m_{\phi}$ less than $1.4 \mathrm{TeV}$ in the hadronic and semileptonic modes, respectively. 


\section{Acknowledgments}

This work was supported by the National Natural Science Foundation of China, under Grants No. 11021092, No. 10975004 and No. 11135003. 
[1] E. Alvarez, L. Da Rold, J. I. S. Vietto, and A. Szynkman, JHEP 1109, 007 (2011), 1107.1473.

[2] U. Haisch and S. Westhoff (2011), * Temporary entry *, 1106.0529.

[3] E. L. Berger, Q.-H. Cao, C.-R. Chen, and H. Zhang, Phys.Rev. D83, 114026 (2011), 1103.3274.

[4] J. Cao, L. Wu, and J. M. Yang, Phys.Rev. D83, 034024 (2011), 1011.5564.

[5] C. Degrande, J.-M. Gerard, C. Grojean, F. Maltoni, and G. Servant, JHEP 03, 125 (2011), 1010.6304 .

[6] M. Battaglia and G. Servant (2010), 1005.4632.

[7] Q.-H. Cao, D. McKeen, J. L. Rosner, G. Shaughnessy, and C. E.M. Wagner, Phys.Rev. D81, 114004 (2010), 1003.3461.

[8] J. Alwall, J. L. Feng, J. Kumar, and S. Su, Phys. Rev. D81, 114027 (2010), 1002.3366.

[9] T. Han, R. Mahbubani, D. G. Walker, and L.-T. Wang, JHEP 0905, 117 (2009), 0803.3820.

[10] V. Barger, T. Han, and D. G.E Walker, Phys.Rev.Lett. 100, 031801 (2008), hep-ph/0612016.

[11] J. Andrea, B. Fuks, and F. Maltoni (2011), 1106.6199.

[12] J. F. Kamenik and J. Zupan (2011), 1107.0623.

[13] Z. Dong, G. Durieux, J.-M. Gerard, T. Han, and F. Maltoni (2011), * Temporary entry *, 1107.3805.

[14] R. Barbier, C. Berat, M. Besancon, M. Chemtob, A. Deandrea, et al., Phys.Rept. 420, 1 (2005), hep-ph/0406039.

[15] S. M. Barr, Phys.Lett. B112, 219 (1982).

[16] A. Aktas et al. (H1), Eur. Phys. J. C36, 425 (2004), hep-ex/0403027.

[17] S. Chekanov et al. (ZEUS), Eur. Phys. J. C50, 269 (2007), hep-ex/0611018.

[18] S. Chakrabarti, M. Guchait, and N. K. Mondal, Phys. Rev. D68, 015005 (2003), hep$\mathrm{ph} / 0301248$.

[19] G. Bhattacharyya, D. Choudhury, and K. Sridhar, Phys. Lett. B355, 193 (1995), hep$\mathrm{ph} / 9504314$.

[20] K. Nakamura et al. (Particle Data Group), J.Phys.G G37, 075021 (2010).

[21] S. Herrlich and U. Nierste, Phys. Rev. D52, 6505 (1995), hep-ph/9507262.

[22] B. de Carlos and P. L. White, Phys. Rev. D55, 4222 (1997), hep-ph/9609443.

[23] P. Slavich, Nucl. Phys. B595, 33 (2001), hep-ph/0008270. 
[24] M. Ciuchini, V. Lubicz, L. Conti, A. Vladikas, A. Donini, et al., JHEP 9810, 008 (1998), erratum added online, Mar/29/2000, hep-ph/9808328.

[25] A. J. Buras (1998), hep-ph/9806471.

[26] A. J. Buras, M. Jamin, and P. H. Weisz, Nucl. Phys. B347, 491 (1990).

[27] S. Herrlich and U. Nierste, Nucl. Phys. B419, 292 (1994), hep-ph/9310311.

[28] J. Urban, F. Krauss, U. Jentschura, and G. Soff, Nucl. Phys. B523, 40 (1998), hep$\mathrm{ph} / 9710245$.

[29] V. Khachatryan et al. (CMS), Phys. Rev. Lett. 105, 211801 (2010), 1010.0203.

[30] G. Aad et al. (ATLAS), Phys. Rev. Lett. 105, 161801 (2010), 1008.2461.

[31] G. Aad et al. (ATLAS), New J. Phys. 13, 053044 (2011), 1103.3864.

[32] S. Chatrchyan et al. (CMS) (2011), 1107.4771.

[33] G. Aad et al. (ATLAS Collaboration) (2011), 1108.6311.

[34] J. Alwall, M. Herquet, F. Maltoni, O. Mattelaer, and T. Stelzer, JHEP 06, 128 (2011), 1106.0522 .

[35] N. D. Christensen and C. Duhr, Comput. Phys. Commun. 180, 1614 (2009), 0806.4194.

[36] G. Belanger, F. Boudjema, A. Cottrant, A. Pukhov, and S. Rosier-Lees, JHEP 0403, 012 (2004), hep-ph/0310037.

[37] G. Aad et al. (ATLAS Collaboration), Phys.Lett. B707, 478 (2012), 1109.2242.

[38] M. L. Mangano, M. Moretti, F. Piccinini, R. Pittau, and A. D. Polosa, JHEP 0307, 001 (2003), hep-ph/0206293.

[39] T. Sjostrand, S. Mrenna, and P. Z. Skands, Comput.Phys.Commun. 178, 852 (2008), 0710.3820 .

[40] T. Sjostrand, S. Mrenna, and P. Z. Skands, JHEP 0605, 026 (2006), hep-ph/0603175.

[41] G. Aad et al. (The ATLAS Collaboration) (2009), 0901.0512. 